Aus dem Allgemeinen Krankenhause Hamburg-Barmbeck.

\title{
Ueber die Behandlung des Morbus Basedowii und der Struma maligna mit Röntgenstrahlen.
}

\author{
Von Prof. Dr. P. Sudeck.
}

Beim echten Morbus Basedowii zeigt die Sehilddrüse statt der kubischen Epithelblase des normalen Organs, welche Kolloidsubstanz einschließt, stark gewucherte Zylinderepithelien. Die Schilddrüse sieht im Mikroskop aus wie ein papilläres Adenom und enthält statt des kolloiden Inhalts nur ein flüssiges Sekret, dem offenbar giftige Eigenschaften innewohnen (Dysthyreoidismus).

Nun handelt es sich zwar hierbei nicht um eine tumorartige, rasche Neubildung junger Zellen, die den Röntgenstrahlen eine so günstige Angriffsfläche bieten, aber sicherlich doch um ein Drüsengewebe, das sich in abnorm lebhafter Funktion befindet, und man sollte von vornherein, da auch die anatomische Lage der Schilddrüse günstig ist, annehmen', da $B$ dieses $G_{2}$ webe durch Röntgenstrahlen zu beeinflussen sei. Diese Erwartung wird in der Tat durch die Erfahrung im ganzen bestätigt.

Auf der einen Seite darf die Bestrahlung nicht zu weit getrieben werden, weil die Zerstörung des gesamten Schilddrüsenepithels mitsamt der Glandula parathyreoidea die Folge sein könnte, anderseits kann man bei der Notwendigkeit dieser Beschränkung kaum etwas anderes von der Röntgenbestrahlung erwarten, als daß die Tätigkeit der Schilddrüsenzellen nur vorübergehend unterdrückt und eingeschränkt wird, um nach der Erholung später wieder einzusetzen. Die Thymusbestrahlung freilich könnte ohne diese Rücksicht vorgenommen werden.

Bei einer Anzahl von Fällen habe ich zum Teil die Schilddrüse, zum Teil den Thymus, zum Teil diese beiden Drüsen bestrahlen lassen. Während einige Fälle sich refraktär erwiesen, konnte ich in anderen eine subjektive und objektive Besserung feststellen. Diese Besserung bleibt aber sowohl an Schnelligkeit als auch an Intensität bei weitem hinter dem gewohnten opera-. tiven Erfolge zurück, vorausgesetzt, daß die Operation genügend ausgiebig gemacht wird.

Die Bestrahlung hat sich mir bewährt als Vorbereitung auf die Operation, besonders in solchen Fällen, an die man sich wegen der Schwere der Erkrankung und der damit bedingten Operationsgefahr nicht heranwagt. Einige solcher Fälle konnte ich nach mehrmonatlicher Vorbehandlung ohne sonderliche Gefahr operieren, andere freilich machten auch dann noch die üblichen schweren Tage nach der Operation durch.

In summa also ist die Bestrahlung ein weniger sicheres und ein weniger wirksames Heilmittel als die Operation, dabei auch nicht ganz ungefährlich, und deshalb kann ich nicht zugeben, daß sie eine der Operation gleichwertige Behandlung genannt werde.

Das Umgekehrte gilt von den malignen Geschwülsten der Schilddrüse. Diese sind in erfreulicher Weise der Bestrahlung zugängig, und zwar in so hohem Maße, daß man die Erfolge nicht nur durch die verhältnismäßig günstige, oberflächliche Lage der Geschwülste zu erklären vermag, sondern man muß den besonderen Charakter der Geschwulstzellen zur Erklärung heranziehen.

Die Struma maligna ist keine einheitliche Geschwulstart, vielmehr zeigt sie einen sehr verschiedenen histologischen Charakter. Es gibt Karżinome verschiedener Art, Sarkome verschiedener Art und Mischgeschwülste (Sarko-Karzinome). Ob ein Unterschied in den einzelnen Geschwulstarten in bezug auf die Reaktion auf die Röntgenstrahlen besteht und welcher, das kann erst durch ausgedehnte Erfahrungen festgestellt werden. Es scheint mir aber, daß sie alle ein besonders dankbares Objekt für die Strahlenbehandlung sind. Von sechs Fällen, die ich be- 
handelt habe, ist ein Fall von Sarko-Karzinom dauernd geheilt, drei Fälle von Karzinomen mit alveolarem Charakter sind lokal gehəilt und an Metastasen zugrundegegangen. Zwei Fälle (mi* kroskopisch nicht bestimmt) sind noch in Behandlung. Der eine von ihnen zeigt eine langsame, der andere eine rapide Verkleinerung.

Ich habe Grund zu der Annahme, daß bei den lokal geheilten Fällen, die an $M$ stastasen gestorben sind, sich bessere Risultate hätten erreichen lassen, wenn die Patienten ihrem L ziden mehr Aufmerksamkeit geschenkt hätten und vor allen Dingen früher in die Behandlung gekommen wären (s. die Krankəngeschichten!).

Umgekıhrt ist für die Chirurgie die Struma maligna ein höchst unerfreuliches Kapitel. Nur selten kommen diese Geschwülste zur Operation, bevor sie die Kapsel der' Schilddrüse durchbrochen und die Umgebung infiltriert haben. Sie pflegen ungemein fest mit der Umgebung verwachsen zu sein. Mir ist es noch nie gelungen, die Operation radikal mit Hoffnung auf Erfolg durchzuführen, und selbṣt wenn es gelingen könnte, den Tumor total zu exstirpieren, so könnte es nicht ohne Totalexstirpation der Schilddrüse geschehen. Außerdem würden die Epithelkörperchen gefährdet werden. Ich habe einen solchen Fall erlebt, der an Tetanie zugrundeging. Vor kurzem konnte ich eine karzinöse Schilddrüse mit guter Aussicht auf Erfolg in toto exstirpieren.

So kommen also von beiden Seiten, von der einen das anatomisch Ungünstige der Verhältnisse, von der anderen die erfreuliche Reaktion auf die Röntgenstrahlen zusammen, um bei der Indikationsstellung der Behandlung ohne weiteres zugunsten der Röntgenbestrahlung zu entscheiden. Ich zähle diese Geschwulstart zu den wenigen, die ich in jedem Falle, ohne den Versuch der chirurgischen Heilung zu machen, von vornherein der Röntgentherapie zuzuweisen für das Richtige halte.

Ich lasse, um das Gesagte anschaulicher zu machen, einen kurzen Auszug aus den Krankengeschichten folgen.

Fall 1. 78jährige Dame, seit längerer Zeit Strumaträgerin, in der letzten Zeit starkes Wachstum bis zu bedeutender Größe mit Dyspnoe, auch während der Ruhe, die zur Operation nötigt.

Operation in Lokalanästhesie. Entfernung der rechten Hälfte. Patientin kollabiert, ist pulslos und reaktionslos. Die Operation muß abgebrochen werden. Das Präparat (Untersuchung durch Prof. Sim. monds) besteht aus einem über faustgroßen Lappen und zwei hühnereigroßen Stücken. Auf dem Durchschnitt mausgraues Gewebe ohne erkennbare Schilddrüsenzeichnung, von weicher Konsistenz, durchsetzt mit mehreren scharf umgrenzten, gelben Herden von Linsen- bis Erbsengröße. Normal erscheinendes Schilddrüsengewebe findet sich nur an einer Stelle in haselnußgroßer Ausdehnung, mikroskopisch Sarkom mit karzinomatösen Partien. Sarko-Karzinom.

Die Patientin erholte sich. Die Dyspnoe ist zunächst verschwunden, mit erneutem Wachstum der Geschwulst kehrt sie wieder. Röntgenbestrahlung durch Herrn Dr. Lorey, darauf Rückgang und völlige Heilung bis zum heutigen Tage. Die jetzt 83 jährige Dame ist gesund und lokal ohne pathologischen Befund. Die Operation liegt 51/2 Jahre zurück.

Fall 2. Etwa 55jähriger Herr. Unregelmäßiger, stark verwachsener Tumor der Schilddrüse, die Drüse übersehreitend und in die Umgebung hineingewachsen, zumal in der Zungenbeingegend ein großer, harter Tumor. Operationsversuch zeigt unüberwindliche technische Schwierigkeiten. Die Operation wird nach Entfernung einer Schilddrüsenhälfte abgebrochen. Das Präparat (Untersuchung von Prof. Si m monds) ist eine gänseeigroße Geschwulst, zum Teil solide, von grauer, körniger Schnittfläche, zum Teil zentral verfallen mit Höh. lungen. Normale Schilddrüse nicht zu erkennen, mikroskopisch AdenoKarzinom.

Röntgenbestrablung (Dr. Haenis ch). Bedeutende Verkleinerung bis zum Verschwinden der Geschwulst, in der Zungenbeingegend bleibt eine, wenn auch bedeutend kleinere, harte Schwellung. Inzision dieser Geschwulst in der Absicht, ein Mesothoriumpräparat direkt in das Geschwulstgewebe einzulegen, dann Probeexzision aus dem Tu mor, lie bei der mikroskopischen Untersuchung durch Prof. Simmonds "nichts Krebsiges" zeigte. Der Tumor zeigt nur narbiges Gewebe, das Sehilddrüsenkarzinom. war lokal ausgeheilt. Dier Patient ging aber an einer Wirbelsäulenmetastase mit Rückenmarkskompression zugrunde. Eine Sektion ist nicht gemacht worden.

Fall 2. 61 jähriger Mann. Seit drei Vierteljahren Halsanschwellung bemerkt, zuerst in der Mitte, dann auch an der rechten Seite. Seit einem halben Jahre bei Anstrengungen Kurzluftigkeit, in den letzten vier Wochen auch in der Ruhe. Linker Schilddrüsenlappen gut hühnereigroß, derbe, harte Konsistenz. Isthmus und rechter Lappen faustgroßer Tumor, bretthart, höckerige Oberfläche. Die rechte Halsseite ist von der Klavikula bis zum Unterkiefer von Tumormassen erfüllt, sodaß keine Halsausbuchtung mehr besteht. Der Kehlköpf nach links verdrängt. Der Tumor ist fest verwachsen, nicht verschieblich und setzt sich unter die Klavikula fort. In beiden Supraklavikulargruben haselnußgroße, harte Drüsen.

Bedeutende Dyspnoe, Patient kann wegen der Kurzluftigkeit nicht flach liegen. Die erste Bestrahlung (Dr. Haenisch) muß wegen Erstickungsanfalls abgebrochen werden. Patient wird für moribund wieder ins Bett gebracht. Fortsetzung der Bestrahlung in sitzender Stellung mit großen Dosen. Nach der zweiten Bestrahlung ist der Tumor kleiner und weicher, nach der ersten Bestrahlungsserie der Hals umfang von 47 auf $39^{1 / 2} \mathrm{~cm}$ zurückgegangen. Nach einer zweiten Serie mit einer Behandlungszeit von sieben Wochen in gutem Heilungsfort schritt entlassen mit der Weisung, nach vier Wochen wiederzukommen.

Da der Patient sich wohl fühlte, befolgte er die Weisung nicht, kommt erst nach einem Jahr wieder mit Metastasen retrosternal in der Lange und multipel in den Knochen. Es gelingt Dr. Haen is ch, sogar die tiefen Lungenmetastasen wenigstens zum Teil fast zum Verschwinden zu bringen. Patient geht zugrunde an zahlreichen Metastasen in den Knochen, im Gehirn und in den Lungen.

Die Schilddrüsengeschwulst war bei klinischer Untersuchung vollständig verschwuñden ohne Ausfallerscheinungen.

Im Sektions protokoll (Prof. Fahr) findet sich über den $\mathrm{Zu}$ stand der Schilddrüse nur der kurze Vermerk: Der rechte Schilddrüsenlappen fehlt. Die Metastasen zeigen mikroskopisch Karzinom von adenomatösem Bau (Prof. Fahr).

Fall 4. 67jährige Frau. Seit $1 \frac{1}{2}$ Jahr Verdickung des Halses bemerkt, die allmählich größer und härter wurde. Kommt wegen Dyspnoe, besonders stark im Liegen. Schilddrüse: rechter Lappen hühnereigroß, derb und verschieblich. Tumor walnußgroß, ebenfalls derb. Linker Lappen kleiner als der rechte, nicht ganz so hart. Der Tumor greift in den Sternokleidomastoideus über. Bestrahlung durch Dr. Haenis ch. Zunächst Vergrößerung des Tumors. Wegen Verdachts einer chronischen Strumitis Operationsversuch; wegen unüberwindlicher Verwachsungen nur Probeexzision. Besonders der Sternokleido war durchwachsen. Bei der Operation bleibt der Zweifel, ob Tumor oder chronische Entzündung.

Anatomische Untersuchung (Prof. Fahr). Struma carcinomatosa. Weitere Röntgenbestrablung kombiniert mit Radiumbestrahlung von der Wunde aus bringt die Geschwulst völlig zum Verschwinden. $71 / 2$ Monate nach der ersten Aufnahme Eintrag in die Krankengeschichte: Die Schilddrüse ist klein und weich, ein pathologischer Befund nicht mehr nachweisbar. 13 Monate nach der Aufnahme Eintrag: Die Schilddrüse kaum fühlbar, nicht empfindlich. Die Patientin ist trotzdem, fast zwei Jahre nach Beginn der Behandlung, an Metastasen zugrunde gegangen.

Auch in diesem Falle gelang es Dr. Haenisch, die röntgeno. logisch nachgewiesenen Lungenmetastasen in weitgehendem Maße zur Einschmelzung zu bringen.

Die Sektion wurde verweigert. 